\title{
Breeding for Heat Tolerance-An Approach Based on Whole-plant Physiology
}

\author{
A.E. Hall \\ Department of Botany and Plant Sciences, University of California, Riverside, CA92521
}

In hot environments, maintenance of plant productivity requires that limiting plant processes are not irreversibly damaged by heat. All plant processes are irreversibly damaged if it is hot enough for a sufficient time. Consequently, in designing breeding programs to incorporate heat tolerance, answers are needed to the following questions:

a) What types of hot weather are occurring in production environments, considering day and night temperatures at different times during the season; and, to what extent are they causing reductions in yield? This information is useful for establishing the priority to be given to breeding heat tolerance into cultivars for different target environments.

b) What stages of plant development and plant processes are most sensitive to high' temperatures and are responsible for thereductions in yield? This information would facilitate the development of efficient techniques for screening germplasm for heat tolerance. It would also be useful to know whether other correlated factors such as evaporative demander day length influence plant response to hot weather.

c) How is heat tolerance inherited, and are there any other characters associated with heat tolerance through genetic linkage or pleiotropy that influence crop adaptation or suitability as a cultivar? This information is useful for developing effective breeding strategies.

I will describe studies that have enabled us to incorporate heat tolerance into cowpea [Vigna unguiculata (L.) Walp.]. The same general approach could be effective with several annual flowering plants, including soybean; cotton, tomato, rice, and sorghum, which have exhibited responses to heat similar to cowpea. We became interested in heat tolerance when we observed negative correlations between grain yield and high temperatures during early flowering for cowpeas sown at different dates (Turk et al., 1980). We suspected that high temperatures were causing excessive flower abscission, because low seed yields were associated with low numbers of pods per peduncle and per unit ground area.

\footnotetext{
This paper was presented as part of the symposium "Plant-Environment Interactions from Subcellular to Plant Community", held at the 83rd ASHS Annual Meeting/XXII International Horticultural Congress, 13 Aug. 1986, Univ. of California, Davis.
}

The types of hot weather and stages of development when cowpeas are seriously affected by high temperatures

By controlled-environment studies, we discovered that high night air temperatures can cause excessive flower abscission (Warrag and Hall, 1984b), whereas plants subjected to high day air temperature or high root-zone temperature exhibited normal levels of flower abscission (Warrag and Hall, 1984a). Flower abscission was greater for plants subjected to both high night and high day temperatures than for plants subjected to just high night temperature, and higher levels of embryo abortion also occurred (Warrag and Hall, 1983). The detrimental effects of high temperature are probably direct and not due to enhanced water stress resulting from higher evaporative demands. High night temperatures should not have much influence on plant water status because stomata are closed at this time, and moderate drought due to soil drying did not influence the level of flower abscission at optimal night temperatures (Warrag and Hall, 1984b).

Artificial pollinations established that the excessive flower abscission and low pod set of plants subjected to high night temperature were due to male sterility (Warrag and Hall, 1984 b). Plants were transferred between growth chambers having optimal and high night temperatures for different durations (Warrag and Hall, 1984b). Analyses of the pulses of flower abscission and pod set that were induced indicated that, for plants grown under optimal night temperatures, the stage of floral development that is sensitive to heat stress occurs $6 \pm 1$ days before flower opening. This corresponded with a stage of development occurring just after the release of tetrads from the microspore mother cell sac. At anthesis, some of the pollen grains from plants grown under high night temperatures were small, with a wrinkled exine and low viability (Mutters, 1988; Warrag and Hall, 1984b). Transfer of pollen to the stigmatic surface (which normally occurs within the same flower in cowpea) was impaired in some cases due to anthers not dehiscing (Mutters, 1988; Warrag and Hall, 1983; Warrag and Hall, 1984b). The factors most responsible for the male sterility have not been elucidated. To facilitate subsequent discussion, the stage in which cowpeas are sensitive to heat during pollen and anther development is called stage 2 .

Contrasting cowpea genotypes have been screened for heat tolerance by sowing them in the Imperial Valley Field Station of the Univ. of California in mid-June (Hall and Pate], 1987; Warrag and Hall, 1983). In this environment, photoperiod--insensitive cowpeas with moderately early maturity begin flowering during late July and early August, when daily maximum and minimum temperatures are extremely high $(39-43 \mathrm{C}$ and 22-26C, respectively) and most genotypes set very few pods (Hall and Patel, 1987; Warrag and Hall, 1983). Two genotypes were discovered, TVu 4552 and Prima, which set many pods in extremey hot conditions, and their tolerance to high temperatures was confirmed by studies in controlled environments (Warrag and Hall, 1983). Observations made on plants under very hot, long-day field conditions (Imperial Valley) and warm-to-hot (35/ 17, day/night), long-day field conditions (Riverside, Calif.) indicated that some cowpea genotypes are sensitive to heat during early floral bud development (stage 1). For these cowpea genotypes, floral bud development is delayed or completely inhibited by very hot, long-day conditions, but not under warm-to-hot, long-day conditions (Dow elmadina and Hall, 1986; Hall and Patel, 1987). Subsequent research in controlled environments demonstrated that sensitivity to heat during floral bud development only occurs in long days and not in short days (Dow elmadina and Hall, 1986; Mutters, 1988). Cowpea genotypes have been separated into eight classes, depending on their relative sensitivities to heat during stages 1 and 2 of floral development, under long-day conditions (Hall and Patel, 1987).

\section{Thermal regimes under which cowpeas are grown and the influence of these temperatures on grain yield}

Substantial differences in night and day air temperatures occur during early flowering in the different regions of the world where cowpea are grown (Hall and Patel, 1987; Nielsen and Hall, 1985). The highest night temperatures occur in the tropics, especially in the Sahelian zone of Africa and also parts of India (Hall and Patel, 1987; Nielsen and Hall, 1985). Substantial differences in night temperature also occur during various summer months and locations in the subtropical and temperate zones where cowpeas are grown in Califorina (Nielsen and Hall, 1985a).

An experimental system was developed to evaluate the influence on cowpea of the night 
temperatures experienced in different parts of the subtropics and tropics (Nielsen and Hall, 1985a). This system involved enclosing field plots at night with insulated plastic sheets; heating the air with fans, heaters, and convection tubing; and controlling temperatures using differential thermostats to dif, ferent levels above ambient conditions. When night air temperatures were raised to levels that occur in the hotter subtropics and tropics for 2 to 4 weeks during early flowering, there were progressive increases in floral abscission and decreases in grain yield with a sensitive cultivar, but the two heat-tolerant cowpea strains exhibited less floral abscission (Nielsen and Hall, 1985b). Apparently, cowpea cultivars with heat tolerance during floral development (stage 2) are needed in the tropics and in the hotter, subtropical areas of California.

\section{Inheritance of heat tolerance in cowpeas and breeding cultivars with improved adaptation to hot environments}

One aspect of the strategy used in this breeding program is the division of a complex process that depends on many genes into subprocesses that are more-simply inherited. This facilitates the use of a backcross breeding program. Many defects are present in the cowpea strains TVu 4552 and Prima, which have substantial heat tolerance. Repeated crosses are needed with parents that can donate appropriate genetic backgrounds. Knowledge of the subprocesses involved in heat tolerance and their inheritance facilitates the choice of recurrent parents. Some potential parents already have some of the genes responsible for heat tolerance, but the phenotype does not have adequate adaptation to hot weather because certain key subprocesses are sensitive to heat. It also facilitates choice of the selection schemes needed to incorporate all of the genes necessary for adaptation to hot weather.

Inheritance studies with $\mathrm{F}_{1}, \mathrm{~F}_{2}, \mathrm{~F}_{3}$, and backcross progenies involving the two heattolerant parents and a heat-sensitive cultivar (CB5) indicate that heat tolerance during floral bud development (stage 1) is conferred by the same recessive gene in $\mathrm{TVu} 4552$ and Prima (P. N., Patel, unpublished data). The inheritance of heat tolerance during pollen and anther development (stage 2) is conferred by a dominant gene, but there is substantial environmentally induced variation in this trait (K.O. Marfo, unpublished data). High day air temperatures can cause embryo abortion and the development of asymmetrical cotyledons (Warrag and Hall, 1983; Warrag and Hall, 1984a). High night temperatures cause undesirable browning of the seedcoat in some cowpea strains (Nielsen and Hall, 1985b). Inheritance studies with $\mathrm{F}_{1}$, $\mathrm{F}_{2}, \mathrm{~F}_{3}$, and backcross progenies involving TVu 4552, which exhibits heat-induced browning of the seedcoat, and CBS and Bambey 21 indicated that this detrimental trait in TVu 4552 is conferred by a dominant gene; no linkage was observed with the genes in $\mathrm{TVu} 4552$ that confer heat tolerance (Patel and Hail, 1988).
The overall breeding strategy conducted by Patel consisted of making simple crosses and backcrosses using TVu 4552 and Prima as sources of heat tolerance during stages 1 and 2, and CB5 and several African cowpeas as sources of heat tolerance during seedcoat development and other desirable agronomic characters for subtropical and tropical zones. $\mathrm{F}_{2}$ and selfed backcross progenies were screened for ability to set pods under extremely high temperatures in the Imperial Valley of California-this procedure would incorporate genes conferring tolerance to heat during stages 1 and 2 and absence of seedcoat browning. In subsequent generations, lines of plants were screened for agronomic characters in an environment representative of warm-to-hot production conditions (Riverside, Calif.). In theory, heat tolerance during stage 1 and seedcoat development should now be fixed in selected lines because they are conferred by recessive genes, but the initial screen in the Imperial Valley is not perfect and heat tolerance during stage 2 involves one or more dominant genes. Also, some temperature variation does occur in field environments, and it is not excessively hot every day in the summer in the Imperial Valley. Consequently, selected lines were screened a second time for heat tolerance in the Imperial Valley 'in the summer, with emphasis on incorporating heat tolerance during stage 2 (pod set). Advanced lines from this breeding program have the capability to set many pods during extremely hot weather in the Imperial Valley, while heat-sensitive parents set very few pods. The four best heat-tolerant lines produced $612 \%$ greater grain yields than the heat-sensitive parent in a yield trial during the summer in the Imperial Valley (Hall and Patel, 1987). Advanced heat-tolerant lines are now being tested in various locations that are representative of different production environments in California to evaluate the impact of incorporating heat tolerance at flowering on productivity under farm conditions.

The field screening procedure and type of heat tolerance during floral bud development available in TVu 4552 and Prima resulted in our simultaneously selecting for heat tolerance during flowering, early flowering, and insensitivity to photoperiod with respect to the timing of flowering. All of these traits are desirable in many cowpea production environments. Where it is desirable to retain photoperiod sensitivity with respect to the timing of flowering, or select for late flowering while selecting for heat tolerance during floral development, it may be necessary to screen in glasshouses where the necessary combinations of night and day temperatures and photoperiod can be achieved. We have evidence indicating that the quality of white light influences the interactive effects of high temperature and day length on floral development (Mutters, 1988). Consequently, where controlled environments are used for screening for heat tolerance, it is necessary to use either natural sunlight or the type of artificial lights that will elicit the same responses as sunlight.

\section{Conclusions}

An example was presented of a breeding program designed for developing cultivars with improved adaptation to hot environments. The approach involves characterizing the following: The target environments where the cultivars will be grown; the types of hot weather, stages of plant growth and plant processes where damage is occurring that is responsible for reductions in yield; and the inheritance of heat tolerance at critical stages. This information was then used to design and execute a conventional backcross breeding program that resulted in the development of cowpea strains with improved productivity in hot environments.

The same general approach could be effective in breeding plants for resistance to other stresses. For research on the effects of stress on individual plant processes to contribute directly to the development of stressresistant plants, it should be established that: 1) the plant process studied is particularly sensitive to stress and is at least partially responsible for the stress-induced reductions in yield that occur on farms and 2) the sensitivity of the plant process can be manipulated genetically without detrimental side effects. Studies at the molecular or cellular level should also follow these guidelines if they are to be directly useful in germplasm improvement. For example, the significance of heat shock proteins for plant adaptation has not been established (Sachs and Ho, 1986). The cowpea germplasm identified and developed by this project, which has tolerance or susceptibility to heat at different stages of floral development, may be useful for testing hypotheses concerning potential physiological roles of specific heat-shock proteins. However, the whole-plant approach used in this project appears to be effective for incorporating heat tolerance, and the need for complementary molecular approaches has not been established.

\section{Literature Cited}

Dow el-madina, I.M. and A.E. Hall. 1986. Flowering of contrasting cowpea [Vigna unguiculata (L.) Walp.] genotypes under different temperatures and photoperiods. Fields Crops Res. 14:87-104.

Hall, A.E. and P.N. Patel. 1987. Cowpea Improvement for Semi-arid Regions of Sub-Saharan Africa. Proc. Intl. Drought Symp. on Food Grain Production in Semi-Arid Regions of SubSaharan Africa. SAFGRAD, Nairobi, Kenya, May 1986.

Mutters, R.G. 1988. Reproductive physiology of cowpea [Vigna unguiculata (L.) Walp.] at high temperatures with different photoperiods. PhD Diss., Univ. Calif., Riverside.

Nielsen, C.L. and A.E. Hall. 1985a. Responses of cowpea [Vigna unguiculata (L.) Walp.] in the field to high night air temperature during flowering: I. Thermal regimes of production regions and field experimental system. Field Crops Res. 10:167-179.

Nielsen, C.L. and A.E. Hall. 1985b. Responses of cowpea [Vigna unguiculata (L.) Walp.] in the field to high night air temperature during flowering: II. Plant responses. Field Crops Res. 10:181-196. 
Patel, P.N. and A.E. Hall. 1988. Inheritance of heat-induced brown discoloration in seed coats of cowpea. Crop Sci. 28:929-932.

Sachs, M.M. and T.-H.D. Ho. 1986. Alteration of gene expression during environmental stress on plants. Annu. Rev. Plant Physiol. 37:363376.

Turk, K. J., A.E. Hall, and C.W. Asbell. 1,980.
Drought adaptation of cowpea. 1. Influence of drought on seed yield. Agron. J. 72:413-420. Warrag, M.O.A. and A.E. Hall. 1983. Reproductive responses of cowpea to heat stress: Genotypic differences in tolerance to heat at flowering. Crop Sci. 22:1088-1092.

Warrag, M.O.A. and A.E. Hall. 1984a. Reproductive responses of cowpea [Vigna unguicu- lata (L.) Walp.] to heat stress: L Responses to soil and day air temperatures. Field Crops Res. 8:3-16.

Warrag, M.O.A. and A.E. Hall. 1984b. Reproductive responses of cowpea [Vigna unguiculata (L.) Walp.] to heat stress: IL Responses to night air temperature. Field Crops Res. 8: 1733 .

\title{
FEATURE
}

\section{Physiological Aspects of the Control of Water Status in Horticultural Crops}

\author{
Hamlyn G. Jones \\ Institute of Horticultural Research, Wellesbourne, Warwick CV35 9EF, United Kingdom
}

Plant water status is arguably the single most-important factor that must be controlled to obtain high yields of good-quality horticultural produce. A clear understanding of how water deficits affect crops and of the natural adaptive processes by which different species respond to limited water availability should enable us to optimize management procedures (e.g., how and when to irrigate) and to breed better cultivars for particular conditions. There have been many reviews in recent years concerned with the techniques of water status measurement; for example, there was a symposium on "Water stress measurement techniques -instrumentation and procedures" at the 81st ASHS Annual Meeting, Vancouver, B.C. (Kanemasu et al., 1985; Spomer, 1985; Syvertsen, 1985), and also another extensive review of techniques for measuring water status (Kirkham, 1985). There has, however, been relatively little emphasis on the relevance of the different methods.

I outline in this paper some of the physical and biological processes involved in the control of plant water status and discuss the choice of water status measure for particular purposes. In particular, my objective is to emphasize some problems, often unrecognized, that may be encountered in water relations experimentation. Failure to take into account some of these factors in the past has resulted in many erroneous conclusions being drawn.

Examples of areas where a clear understanding of the mechanisms involved in the control of plant water status is important include: the measurement of water status; breeding new cultivars; design of experiments for the field, controlled environments, or glasshouses; and understanding the role of stomata.

\section{CONTROL OF PLANT WATER} STATUS: BASIC PRINCIPLES

The main processes involved in the con-

This paper was presented as part of the symposium "Plant-Environment Interactions from Subcellular to Plant Community", held at the 83rd ASHS Annual Meeting/XXII International Horticultural Congress, 13 Aug. 1986, Univ. of California, Davis.

HortSCIEnce, Vol. 25(1), JANUARy 1990 trol of plant water status have been extensively reviewed elsewhere (Jones, 1983a; Kramer, 1983; Slatyer, 1967), with some reviews specifically concerned with horticultural crops (Chalmers et al., 1983; Jones et al., 1985; Kriedemann and Barrs, 1981). The complex series of environmental and physiological controls and some of the interactions between them are summarized in Fig. 1. There are at least four main groups of processes.

a) The soil water potential and those factors involved in its control, including past rainfall and water use, soil texture and depth, and root distribution and activity.

b) Crop evaporation rate, both historic and current (with the transpiration component being especially important in the latter case). As is clear from Fig. 1, there are many environmental factors (e.g., radiation, humidity, and windspeed) as well as physiological factors (e.g., leaf area and display and stomatal conductance) involved.

c) Hydraulic conductance in the flow pathways within the plant are important for controlling the instantaneous values of water potential at any point in the plant. During steady-state conditions, resistances (the inverse of conductance) determine the potential drop across any segment of the pathway according to:

$$
\psi_{2}=\psi_{1}-\mathrm{J}_{\mathrm{v}} \mathrm{R}_{1,2}=\psi_{1}-\mathrm{J}_{\mathrm{v}} / \mathrm{L}_{1,2}[1]
$$

where $\mathrm{J}_{\mathrm{v}}$ is the volume flux density, $\mathrm{R}$ is the hydraulic resistance, $\mathrm{L}$ is the corresponding hydraulic conductance, and subscripts 1 and 2 refer to positions on the pathway. When, however, flow is varying, as, for example, when clouds pass overhead, it becomes necessary for prediction of $\psi_{\text {leaf }}$ to use a dynamic model that includes the capacitances of different parts of the system (Jones, 1978, 1983a; Powell and Thorpe, 1977). Many plants can be approximated by a single resistance and capacitance, so that the time dependence of $\psi_{\text {leaf }}$ after a step change in evaporation rate (E) is given by (Jones, 1983a):

$$
\psi_{\text {leaf }}=A+B \exp (-t / \tau),
$$

where: $A=\left(\psi_{\text {soil }}-E R_{\text {plant }}\right) /\left(R_{\text {plant }} C\right), B=$ $\psi_{\text {initial }}+R_{\text {plant }} E-\left(\psi_{\text {soil }} / R_{\text {plant }} C\right), \tau=R_{\text {plant }} C$ $=$ time constant.

It should be noted that, although flow rates (and corresponding resistances and conductance) are expressed on an area basis, the choice of area basis used, whether it is stem area, xylem area, leaf area, or even ground area, is arbitrary. However, it is usual to avoid problems raised by the complexity of the pathway and to choose either leaf area or ground area.

Factors controlling plant water status

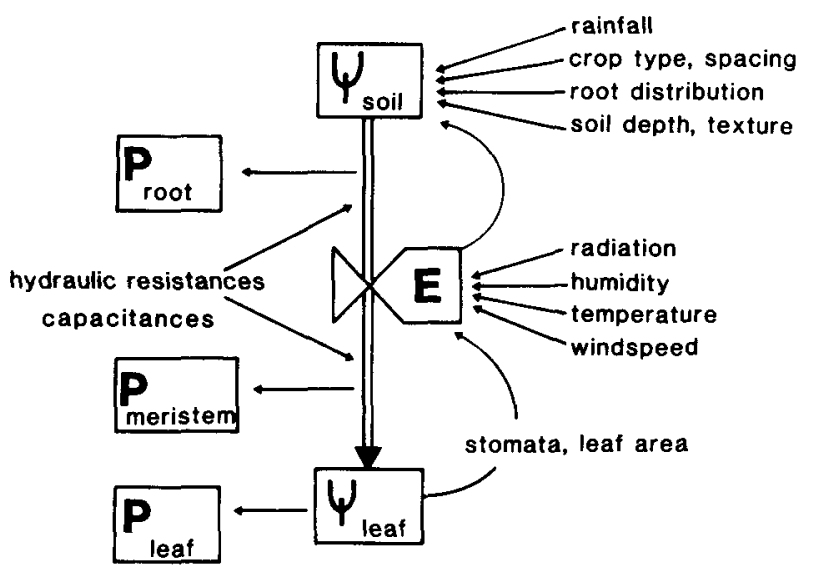

Fig. 1. Processes involved in the control of plant water status. 